DOI: https://doi.org/10.46296/yc.v6i10.0149

\title{
HERRAMIENTAS TECNOLÓGICAS Y DIDÁCTICAS EN EL APRENDIZAJE DE LOS VALORES CÍVICOS EN LOS ESTUDIANTES DE LA UNIDAD EDUCATIVA GUARANDA 43 DE LA PARROQUIA CHIRIJOS DEL CANTÓN PORTOVIEJO
}

\section{TECHNOLOGICAL AND DIDACTIC TOOLS IN THE LEARNING OF CIVIC VALUES IN THE STUDENTS OF THE GUARANDA 43 EDUCATIONAL UNIT OF THE CHIRIJOS PARISH OF THE PORTOVIEJO CANTON}

\author{
Alcívar-Loor Ramón Aparicio "; Molina-Villacis Paulina de las Mercedes ${ }^{2}$ \\ ${ }^{1}$ Maestría en Educación, Mención Educación y Creatividad, Universidad San Gregorio de \\ Portoviejo, USGP. Portoviejo, Ecuador. Correo: e.raalcivar@sangregorio.edu.ec. \\ ${ }^{2}$ Maestría en Educación, Mención Educación y Creatividad, Universidad San Gregorio de \\ Portoviejo, USGP. Portoviejo, Ecuador. Correo: pmolina@sangregorio.edu.ec.
}

\begin{abstract}
Resumen
Las herramientas tecnológicas y didácticas en el aprendizaje de la educación han permitido el abordaje de los contenidos para responder de manera pertinente a las necesidades de aprendizaje estudiantil. Por esta razón, el presente trabajo tiene como objetivo determinar el manejo de las herramientas tecnológicas y didácticas en el aprendizaje de los valores cívicos en los docentes y estudiantes en la Unidad Educativa Guaranda 43 Portoviejo -Ecuador. Metodológicamente se define como una investigación descriptiva, exploratoria y bibliográfica. Para la discusión y análisis de los resultados se utilizaron los métodos teóricos de análisis, inducción y deducción. El instrumento utilizado fue una encuesta electrónica para recaudar la información. Participaron 44 estudiantes y 16 docentes. Los problemas que más se evidenciaron en la investigación fue la poca utilización y manejo de las herramientas tecnológicas por la situación socio económica de los estudiantes y la falta de conectividad en los sectores de vivienda. Los resultados evidenciaron que el manejo de las herramientas tecnológicas y didácticas en el aprendizaje de los valores cívicos presentan complicaciones, debido a la poca utilización de las herramientas tecnológicas educativas de parte de los docentes y estudiantes. Se concluye que, si bien los estudiantes no cuentan con el manejo de herramientas tecnológicas, existe gran aceptación sobre el uso de herramientas educativas básicas, conllevando a mejoramiento del aprendizaje y estrategias didácticas en el alumnado. Los docentes deben enfatizar diversas alternativas didácticas con la finalidad que los estudiantes sigan aplicando los valores cívicos a través de las herramientas tecnológicas educativas, independientemente del área de estudio.
\end{abstract}

Palabras claves: Aprendizaje, herramientas tecnológicas, tecnología, valores cívicos.

\begin{abstract}
The technological and didactical tools in learning education have got the opportunity to reach contents that answer the best way to the needs to students learning process. That's why the goal of this project is to determine the management of the technological and didactic tools. That is to say the learning of civic values of teachers and students of the Unidad Educativa Guaranda 43 from Portoviejo - Ecuador. Its methodology is called a descriptive, explorer and bibliographic investigation. To discus and analyze the results we used the deductive and inductive and analyses methods. It was used an electronic questionnaire in order to gather information. There were forty-four students and sixteen teachers in the investigation. The most evident problems were the lack of using and managing of technological tools. Because of the economic situation of students, because they are not always able to access to internet connection in their neighborhoods. That's why they cannot use those tools and technological devices, even they are
\end{abstract}

Información del manuscrito:

Fecha de recepción: 08 de noviembre de 2021.

Fecha de aceptación: 27 de diciembre de 2021.

Fecha de publicación: 31 de enero de 2022. 
basic ones at the moment. In despite of these problems, they are interested in learning how to use them and to introduce them in new processes of learning, in order to open new opportunities for both teachers and students. Teachers must emphasize civic and learning values for the students to learn them apart of the subject of studies areas.

Keywords: learning, technologic tools, students, technology, civic values.

\section{Introducción}

Las herramientas tecnológicas se han convertido en la actualidad en una herramienta básica para la ejecución de diversos trabajos públicos, entre ellos enfatizando la educación, ya que ha permito desde mucho tiempo atrás el descubrimiento de muchos estudios que han sido de gran ayuda y aporte en diversos aspectos de la ciencia, además, hoy en día sigue ayudando a muchos docentes y alumnos a adaptarse a esta nueva modalidad en aplicar las diversas herramientas educativas.

El desarrollo de las nuevas tecnologías de información y comunicación ha generado diferentes herramientas digitales como son los programas computacionales, redes sociales, entornos virtuales de aprendizaje (EVA), entornos personales de aprendizaje (PLE), gestores de referencias bibliográficas mismos que han propiciado escenarios digitales de trabajo bidireccional entre los usuarios, docente, estudiante, investigador, profesional y otros. Los centros de enseñanza superior no están exentos del empleo de estas tecnologías digitales, la aplicabilidad de estas herramientas tecnológicas está relacionado con los procesos educativos, de investigación, profesional, lúdico, gamificación y otros. (Vargas, 2019, p. 88).

La evolución constante de las herramientas tecnológicas en los últimos años ha impactado considerablemente la forma en la que concebimos la manera de comunicarnos y la forma de acceder a la información en nuestra vida social y académica. "Los medios digitales actuales permiten que los individuos entren en contacto con la información digital y su percepción del mundo cambia de manera considerable en función de lo que viven y reciben en los dispositivos 
tecnológicos actuales" (Trejo, 2018, p. 617).

En contexto general las herramientas tecnológicas forman parte del gran mundo de la tecnología, hoy en día denominado TIC, siendo de gran aporte para la enseñanza y aprendizaje de los estudiantes y docentes.

Dentro de las posibilidades que la incorporación de las tecnologías de la información y la comunicación (TIC) ofrece a la educación se encuentra la atención a la diversidad, ya que por medio de estas herramientas se puede facilitar el acceso y la educación de calidad a los estudiantes con diversas necesidades educativas especiales (Romero et al., 2018, p. 85).

En el contexto educativo se estructura la formación de los niños de edad escolar por medio de lineamientos y estándares curriculares que fundamentan las áreas básicas de aprendizaje como son de lengua castellana, inglés, matemática, ciencias naturales, ciencias sociales, tecnología, educación física, artes, civismo, entre otras. Cabe resaltar que estas asignaturas se relacionan en cada periodo académico con la finalidad de que el proceso de enseñanza y aprendizaje beneficie al estudiante. "Es así como las estrategias de enseñanza orientadas por el docente configuran en gran medida el aprendizaje y la relación que establece el estudiante con los contenidos y temáticas que le permiten generar conocimientos a lo largo de su vida" (Pamploma et al., 2019, p. 19).

Una estrategia didáctica es más que solo aplicar una técnica con un listado de actividades o tareas para llevar a cabo, (Mansilla y Beltrán, 2013, p. 29) la definen de la siguiente manera: "la estrategia didáctica se concibe como la estructura de actividad en la que se hacen reales los objetivos y contenidos". Esta estructura implica un proceso que nace desde un punto de partida, que son los contenidos de información, puede ser nueva o alguna información previa que las personas participantes ya posean sobre el tema; "el punto en que se espera llegar al objetivo es con el cumplimiento de lo que se desea alcanzar cuando se propone el 
desarrollo de una estrategia" (Orellana, 2017, p. 136).

Los profesores utilizan estrategias didácticas para desarrollar el contenido de un programa y convertirlo en un concepto significativo. Este proceso se denomina transposición didáctica porque es la herramienta que permite una transferencia didáctica de información.

Las estrategias están enfocadas a cumplir los objetivos que se plantean en un determinado contexto de enseñanza y aprendizaje, donde las estrategias de enseñanza y las de aprendizaje se ponen en práctica. "Las estrategias de enseñanza fomentan las instancias de aprendizaje, promoviendo la participación de los estudiantes, así mismo, las estrategias de aprendizaje, es relevante mencionar que los estudiantes las utilizan para organizar y comprender contenidos o ideas clave" (Flores et al., 2017, p. 13).

Por otra parte, el investigador Pastor, (2019) señala que las estrategias didácticas no se limitan a los métodos y metodologías en las que se enseñan, sino que también incluyen medidas que tienen en cuenta el repertorio de procedimientos, técnicas y habilidades que los estudiantes necesitan aprender; que, como expresa el autor mencionado anteriormente, es un concepto que se corresponde mejor con las tendencias actuales en didáctica.

Los valores cívicos son los comportamientos que se consideran positivos para el desarrollo de la sociedad. Generalmente son reconocidos por los diversos grupos sociales y comunidades repartidos por todo el mundo (Morales, 2019).

Los valores cívicos se establecen en el proceso de adquisición y expresión de cualidades morales, las cuales se adquieren por modelos de convivencia y se manifiestan en la experiencia cotidiana del individuo, pues la imitación desde las edades más tempranas es uno de los mecanismos básicos de la comunicación.

Así, sobre la base del conocimiento y la exigencia de las normas morales vigentes en la sociedad, se va estableciendo una relación de progresiva aceptación hacia ellas, de su 
incorporación a la conducta, de sentimientos de necesidad de estas formas de ser, de jerarquizarlas sobre otras, de su inclusión en aspectos internos de especial significación personal. (Aranda et al., 2017, p. 1031)

\section{Metodología}

El enfoque de la investigación fue de forma cualitativa - cuantitativa y de tipo exploratoria, descriptiva y bibliográfica. Para el proceso de selección de la muestra se utilizó el método Probabilístico de tipo aleatorio y simple, aplicando la fórmula $n=\frac{\mathrm{m}}{\mathrm{a} 2(\mathrm{~m}-1)+1}$ con una muestra de 44 estudiantes de nivel básico de diferentes paralelos y grados (octavo, noveno y décimo) y 16 docentes titulares con un rango de edad entre 32 y 60 años. Para la selección de los docentes se establecieron los siguientes criterios: que su ingreso a la institución fuera a través de concurso de mérito y oposición, que tuvieran mínimo tres años de experiencia docente y que fueran de diferentes disciplinas. La información primaria se obtuvo a través de encuestas utilizando un formulario en Google Form, entrevista online, el bosquejo de información secundaria se recopiló a través de fuentes bibliográficas y para sistematizar y analizar la información recaudada se utilizaron métodos estadísticos.

\section{Resultados y discusión}

Por medio de la encuesta realizada a estudiantes y a docentes del nivel básico de la Unidad Educativa 43 de la parroquia Chirijos, a través del formulario Google Form, se pudo analizar al momento de revisar las encuestas dirigidas a los 16 docentes de la institución, que ellos sí han realizado algún curso sobre manejo en herramientas educativas, pero, consideran que los estudiantes no tienen mucho conocimiento sobre las herramientas tecnológicas. Como se puede observar en la Tabla 1 , que indaga a los docentes sobre cuáles son las herramientas tecnológicas que utiliza con mayor frecuencia. 
Tabla 1. ¿Cuáles de las siguientes herramientas tecnológicas es la que utiliza con mayor frecuencia?

\begin{tabular}{|c|c|c|}
\hline VARIANTES & DOCENTES & PORCENTAJE $(\%)$ \\
\hline Genially & 0 & 0 \\
\hline Symbaloo & 0 & 0 \\
\hline Google Drive & 5 & 31.25 \\
\hline Microsoft Teams & 10 & 62,5 \\
\hline Otro & 1 & 6.25 \\
\hline Total & $\mathbf{1 6}$ & $\mathbf{1 0 0}$ \\
\hline
\end{tabular}

Fuente: Docentes de la Unidad Educativa Guaranda 43 Elaboración: Propia.

Ante las condiciones de la pandemia del COVID 19 las herramientas de mayor uso por parte de los docentes fue Microsoft Teams (63\%), seguida de Google Drive (31.25\%), y el $6.25 \%$, lo que demuestra que los docentes si utilizan las herramientas tecnológicas, pero al dialogar con ellos manifestaron que lo manejan de forma básica. Así mismo, los porcentajes obtenidos en las respuestas de los estudiantes reflejaron coincidencias con los docentes: $61.4 \%$ Microsoft Teams, $34.1 \%$ Google Drive y $4.5 \%$ Symbaloo, lo que indica que también tienen conocimiento de las herramientas tecnológicas, sin embargo, no todos la utilizan de forma correcta; como puede observarse en la tabla 2.

Tabla 2. ¿Seleccione la herramienta tecnológica que haya hecho uso en algún momento?

\begin{tabular}{|c|c|c|}
\hline VARIANTES & ESTUDIANTES & PORCENTAJE (\%) \\
\hline Genially & 0 & 0 \\
\hline Symbaloo & 2 & 4.5 \\
\hline Google Drive & 15 & 34.1 \\
\hline Microsoft Teams & 27 & 61,4 \\
\hline Total & $\mathbf{4 4}$ & $\mathbf{1 0 0}$ \\
\hline
\end{tabular}

Fuente: Estudiantes de la Unidad Educativa Guaranda 43.

Elaboración: Propia. 
Si bien los docentes señalan que han manejado algunas herramientas como Microsoft Teams y Google Drive, en diálogo con ellos vía online manifestaron que les cuesta manejarlo, porque es una temática nueva, y que no cuentan con el dominio de la misma.

Tabla 3. Con que frecuencia hace prevalecer los valores cívicos al momento de hacer uso de alguna herramienta tecnológica.

\begin{tabular}{|c|c|c|}
\hline VARIANTES & DOCENTES & PORCENTAJE (\%) \\
\hline Frecuentemente & 7 & 43.8 \\
\hline Muy Frecuentemente & 6 & 37.5 \\
\hline Poco frecuente & 3 & 18.8 \\
\hline Nada frecuente & 0 & 0 \\
\hline Total & $\mathbf{1 6}$ & $\mathbf{1 0 0}$ \\
\hline
\end{tabular}

Fuente: Docentes de la Unidad Educativa Guaranda 43.

Elaboración: Propia.

Los docentes hacen prevalecer los valores cívicos a través de las herramientas tecnológicas en un $43.8 \%$ frecuentemente y un $18.8 \%$ poco frecuente. Por lo tanto, si se puede prevalecer los valores cívicos a través de las herramientas tecnológicas. "Es un hecho que, en la actualidad, nuestra vida, sociedad y actividades diarias están ligadas y giran en torno a las nuevas tecnologías de la información y comunicación e innovación tecnológica" (Avendaño, 2015).

Tabla 4. Consideras que los docentes puedan observar el uso de tus valores cívicos a través de la utilización de herramientas tecnológicas.

\begin{tabular}{|c|c|c|}
\hline VARIANTES & ESTUDIANTES & PORCENTAJE (\%) \\
\hline Mucho & 25 & 56.9 \\
\hline Suficiente & 13 & 29.5 \\
\hline Poco & 3 & 6.8 \\
\hline Muy poco (nada) & 3 & 6.8 \\
\hline Total & $\mathbf{4 4}$ & $\mathbf{1 0 0}$ \\
\hline
\end{tabular}

Fuente: Estudiantes de la Unidad Educativa Guaranda 43 Elaboración: Propia.

El $56.9 \%$ de los estudiantes consideran que los docentes si pueden observar el comportamiento de sus valores, mientras que el $6.8 \%$ manifiesta que no pueden analizar sus valores cívicos. Estos resultados 
manifiestan que la mayoría de los estudiantes son consciente de que los docentes pueden analizar el comportamiento de los valores cívicos a través del uso de las herramientas tecnológicas. Los documentos curriculares añaden: "La convivencia entre los individuos y entre los grupos que conforman una sociedad pluralista solo es posible en el marco de un conjunto básico de valores compartidos" (Ministerio de Educación, 2020).

\section{Conclusiones}

Las herramientas tecnológicas que con mayor frecuencia utilizan los docentes al momento de implementar sus clases cotidianas es la de Microsoft Teams y la de Google Drive. A su vez, los catedráticos consideran que los valores cívicos sólo deben ser indagados por los docentes de ciencias sociales.

Se pudo evidenciar que tanto a los estudiantes y docentes se les complica el desarrollo de sus clases en la modalidad virtual debido a la falta de garantías para el acceso a una conexión estable de Internet y por la falta de experticia en el manejo de herramientas tecnológicas.

Por último, se define a través de la información recopilada que existe la disposición de docentes y estudiantes de adquirir competencias digitales que propicien el proceso de enseñanza aprendizaje para transmitir los valores cívicos por medio de la incorporación de herramientas tecnológicas., esto se ve evidenciado ya que al encuestar a los estudiantes ellos manifestaron que sus docentes si podrán observar sus valores cívicos, debido a que los estudiantes vienen de una cultura donde aún sus padres priorizan los valores en la enseñanza de sus niños, haciendo que sea más fácil intervenir en ellos. Sin embargo, es necesario que los docentes no dejen de reformar la enseñanza de los valores cívicos y su importancia de aplicarlos al momento de su vida diaria y en el futuro cuando sean grandes profesionales. 


\section{Bibliografía}

Aranda, B., Domínguez, I., Ramos, G., Ávila, Y., Y Durán, D. (2017). El proceso de formación de los jóvenes universitarios en la concepción de educación de género y valores cívicos. Medisan, 8, 1027-1037. http://scielo.sld.cu/scielo.php ?pid=S1029$30192017000800009 \&$ script $=$ sci_arttext\&tlng=pt

Avendaño, V. (2015). Implementación y uso escolar de las tecnologías de la información la comunicación. Mexico.

Barreno, C. (2019). La interculturalidad en el proceso de enseñanza-aprendizaje en la unidad educativa Abdón calderón en el período 20162017.

http://repositorio.utc.edu.ec/bi tstream/27000/5870/1/PI001156.pdf

Casares, L. (2017). Tratamiento de la educación para el consumo en el alumnado de 10 a 16 años escolarizado en centros educativos de la Comunidad Autónoma de Extremadura. https://dehesa.unex.es/flexpa per/template.html?path=https ://dehesa.unex.es/bitstream/1 0662/6302/1/TDUEX_2017 Casares_Avila.pdf\#page $=1$
Díaz, J., \& Rodríguez, M. (2020). El desarrollo local en el presente: Un estado del arte sobre la producción del conocimiento durante la década 2010 - 2020. https://ciencia.lasalle.edu.co/ maest_gestion_desarrollo/18 $0 /$

Espinoza, E., \& Villamar, G. (2019). Las herramientas office en la calidad del aprendizaje significativo.

http://repositorio.ug.edu.ec/bit stream/redug/45046/1/BFILO -PD-INF1-19-024.pdf

Flores, J., Ávila, J., Rojas, C., Sáez, F., Acosta, R., Y Díaz, C. (2017). ESTRATEGIAS DIDÁCTICAS PARA EL APRENDIZAJE SIGNIFICATIVO EN CONTEXTOS UNIVERSITARIOS

(Concepción, Chile ed.). http://docencia.udec.cl/unidd/i mages/stories/contenido/mat erial_apoyo/ESTRATEGIAS \%20DIDACTICAS.pdf

Jaramillo, S. (2020). La educación integral en valores como una forma de prevenir el delito $y$ abolir la pena. https://repositorio.uasb.edu.e c/bitstream/10644/7231/1/T3 126-MDPE-JaramilloLa\%20educacion.pdf

Luz, F. (2020). Directivos docentes resilientes, una propuesta para enfrentar la realidad 
educativa al interior de las instituciones educativas oficiales del municipio de Bucaramanga, Colombia. https://repositorio.umecit.edu. pa/handle/001/2934

Ministerio de Educación. (2020). "Fortalecemos Valores, Principios y Virtudes". Obtenido de CUADERNOS DE FORMACIÓN CONTINUA.:

https://www.unodc.org/docum ents/bolivia/Cuaderno_de_Fo rmacion_Continua_1.pdf

Molinero, M. D. C., \& Chávez, U. (2019). Herramientas tecnológicas en el proceso de enseñanza-aprendizaje en estudiantes de educación superior. 10. http://www.scielo.org.mx/sciel o.php?script=sci_arttext\&pid= S2007-74672019000200005

Morales, A. (2019). Qué son los valores cívicos. https://www.significados.com/ valores-civicos/

Orellana, C. (2017). La estrategia didáctica y su uso dentro del proceso de enseñanza y aprendizaje en el contexto de las bibliotecas escolares. ECiencias de la Información, 134-154, 134-154.

Palacios, J., \& Barreto, G. (2021). Breve análisis de los métodos empleados en la enseñanza de la historia en educación básica. Sociedad \&
Tecnología, 4, 65-73. http://institutojubones.edu.ec/ ojs/index.php/societec/article/ view/77/360

Pamploma Raigosa, J., Saldarriaga, J. C., Y Cano Cuesta, V. (2019). Estrategias de enseñanza del docente en las áreas básicas: una mirada al aprendizaje escolar. Eleuthera, $21, \quad 13$. https://revistasojs.ucaldas.ed u.co/index.php/eleuthera/artic le/view/2221/2127

Pastor, R. (2019). HERRAMIENTAS DIDÁCTICAS ORIENTADAS AL ESTUDIANTE $Y$ EL RENDIMIENTO ACADÉMICO. https://repositorio.upch.edu.p e/bitstream/handle/20.500.12 866/7310/Herramientas_Past orArmendariz_Roberto.pdf?s equence $=1$

Reascos, P., \& Granda, A. (2020). Factores potenciadores de valores cívicos, patrióticos e interculturales en la enseñanza básica. Sociedad \& Tecnología, 3, 42-50. http://institutojubones.edu.ec/ ojs/index.php/societec/article/ view/66/422

Romero Martínez, S. J., Gonzáles, I., García, A., Y Lozano, A. (2018). Herramientas tecnológicas para la educación inclusiva. Tecnología, Ciencia y Educación, 9, 83 - 11. https://udimundus.udima.es/b 
itstream/handle/20.500.12226

/54/Herramientas\%20tecnol\% c3\%b3gicas\%20para\%20la\% 20 educaci\%c3\%b3n\%20inclu siva.pdf?sequence $=1$ \&isAllo wed $=\mathrm{y}$

Rodríguez, A., \& Fernández, R. (2019). ESTUDIO DE CASO EN UNA UNIDAD DE ANALISIS EDUCACIONAL PARA EL TRATAMIENTO AL CONTENIDO DISCIPLINA SOCIAL EN EL PROCESO DE ENSEÑANZAAPRENDIZAJE DE LA EDUCACIÓN CIVICA.

Didasc@ lia: didáctica y educación ISSN 2224-2643, 10 , 264-282. http://revistas.ult.edu.cu/index .php/didascalia/article/view/9 26/923

Trejo Gonzáles, H. (2018). Herramientas tecnológicas para el diseño de materiales visuales en entornos educativos. Sincronía, 74, 617-669.

https://www.redalyc.org/jatsR epo/5138/513855742031/513 855742031.pdf

Vargas Murillo, G. (2019). Competencias digitales y su integración con herramientas tecnológicas en educación superior. Cuadernos Hospital de clínicas, 60, 88-94. http://www.scielo.org.bo/sciel o.php?pid=S1652-

$67762019000100013 \&$ script $=$ sci_arttext
Wilson, A., \& Fernández, R. (2019). Didasc@ lia: didáctica y educación ISSN 2224-2643, 10 , 264-282. http://revistas.ult.edu.cu/index .php/didascalia/article/view/9 26/923

Yagüé, L. (2018). El método lúdico para la formación de valores ético-cívicos en secundaria básica. 14, 106-111. http://scielo.sld.cu/scielo.php ?script=sci_arttext\&pid=S199 0-86442018000500106 